\title{
Biological Activity of Selected Compounds from Annona muricata Seed as Antibreast Cancer Agents: Theoretical Study
}

\author{
Abel Kolawole Oyebamiji $\mathbb{D}^{1},{ }^{1,2}$ Gideon Femi Tolufashe $\mathbb{i}^{3},^{3}$ \\ Olubukola Monisola Oyawoye $\mathbb{D},{ }^{4}$ Temitope A. Oyedepo $\mathbb{D},{ }^{5}$ and Banjo Semire $\mathbb{D}^{2}$ \\ ${ }^{1}$ Department of Basic Sciences, Adeleke University, P.M.B. 250, Ede, Osun State, Nigeria \\ ${ }^{2}$ Computational Chemistry Laboratory, Department of Pure and Applied Chemistry, Ladoke Akintola University of Technology, \\ P.M.B. 4000, Ogbomoso, Oyo State, Nigeria \\ ${ }^{3}$ Department of Chemistry and Biochemistry, Faculty of Sciences, University of Porto, 4169-007 Porto, Portugal \\ ${ }^{4}$ Department of Microbiology, Laboratory of Molecular of Biology, Immunology and Bioinformatics, Adeleke University, \\ P.M.B. 250, Ede, Osun State, Nigeria \\ ${ }^{5}$ Department of Biochemistry, Adeleke University, P.M.B. 250, Ede, Osun State, Nigeria
}

Correspondence should be addressed to Abel Kolawole Oyebamiji; abeloyebamiji@gmail.com

Received 6 August 2020; Revised 17 October 2020; Accepted 24 October 2020; Published 9 November 2020

Academic Editor: Florent Barbault

Copyright (C) 2020 Abel Kolawole Oyebamiji et al. This is an open access article distributed under the Creative Commons Attribution License, which permits unrestricted use, distribution, and reproduction in any medium, provided the original work is properly cited.

\begin{abstract}
Several natural products have been of help to humans, and its effect is noticeable in the medicinal world. Soursop with botanical name Annona muricata L. possesses antidiarrhea, anticold fever, antirheumatism, and antineuralgia properties. In this work, five selected molecular compounds were studied against type 3 of $3 \alpha$-hydroxysteroid dehydrogenase ( $3 \alpha$-HSD). Its anticancer activity was investigated using the quantum chemical method via Spartan 14 software, molecular docking via Discovery studio 2017, AutoDock Tool 1.5.6, AutoDock Vina 1.1.2, and PyMol 1.7.4.4 and the molecular dynamic simulation method via AMBER14 molecular dynamics package. Many descriptors $\left(E_{\mathrm{HOMO}}, E_{\mathrm{LUMO}}\right.$, dipole moment, energy bandgap, area, volume, polarizability, polar surface area, $\log P$, hydrogen bond donor, and hydrogen bond acceptor) which describe the anticancer activity of the studied compounds were obtained. Also, the docking study revealed the inhibiting ability of the studied compound, and it was observed that compound $\mathbf{C}$ possesses a greater ability to inhibit than other studied compounds as well as the standard (5FU).
\end{abstract}

\section{Introduction}

The use of natural product compounds in supporting human health remains one of the vital ways of treatment globally [1]. Phytochemical compounds present in vegetation have played a crucial role in drug discovery, and their cytotoxicity has drawn the attention of researchers over the years [2]. Also, several scientists have declared plants with efficient biological importance to be source for well-being conservation [3]. As reported by Ko and Moon, the use of natural product in treating breast cancer is beneficial, and the chemical compounds present in these products have little or no negative effect compared to synthetic drug molecules [4].

Soursop with botanical name Annona muricata L. belongs to the family Annonaceae, and it is a tropical fruit-bearing tree usually found in some areas of Africa, America, Australia, and Asia [5]. It is a tree with thick leaves that are oblate. Annona muricata possesses touchy, dark green fruit $[6,7]$. Several researchers have reported this plant to be very useful in treating many diseases such as cancer, diabetes, inflammation, and hypertension [8]. According to Vijayameena et al., it helps in curing diabetic due to hypoglycemic and antioxidant features it contains [9]. Most parts of Annona muricata are useful in herbal medicine. Some parts, such as roots and stems, help in lowering blood pressure and also act as an antispasmodic agent [5]. Also, Annona muricata fruit plays a serious role in the medicinal world such as antidiarrhoea, anticold fever, antirheumatism, and antineuralgia and its consumption helps in amassing mother's milk after giving 
birth [10]. As reported by some researchers, the biological activities of extracts from Annona muricata were observed to be very toxic to cancer cells than normal human cells [11-15]. Also, as reported by Shashanka and Devananda, most of the molecular compounds present in this plant have biomedical significance, and further study on this work is still ongoing [16]. According to Rieser et al., it was observed that muricatacin, as well as other compounds present in Annona muricata, possesses antiproliferative characteristics against breast, colorectal, and lung cancer cell line [17]. Wu et al. reported antilung cancer, antibreast cancer, and anticolon cancer activities of annomuricin A and annomuricin B. It was shown that they are potent against the breast, lung, and colon cancer [18]. Moreover, breast cancer has caused the death of several women over the years [19]. The rate at which this malignant neoplasia increases is on the high side [20], and as reported by Keogh et al., almost 95\% of women have moderately growing risk of breast cancer [21]. The prospective methods for curing cancer remain chemotherapy, surgery, and radiotherapy; these methods have been used for several years ago with victory in several cases. Nevertheless, these methods have been recorded futile in some cases such as unconventional metastatic breast cancer [22]. More so, the rate of survival among the patient with breast cancer keeps increasing, and this could be attributed to improvement in drug designing and development [23].

$3 \alpha$-HSD as a vital enzyme plays a serious role in breaking down of androgen [24]. In sex, $3 \alpha$-HSD has been recognised in human to be a serious enzyme due to the role it played [25]. According to Aka et al., some estrogens like 17 $\beta$ oestradiol ignite proliferation of breast cancer cells in woman although $5 \alpha$-dihydrotestosterone ( $5 \alpha$-DHT) have the strength to shrink the growth of breast cancer in woman [26]; as reported by Penning et al., $3 \alpha$-HSD can hinder $5 \alpha$ dihydrotestosterone, which will thereby increase the growth of breast cancer cells in woman [27]. Also, Bo et al. revealed that overriding $3 \alpha$-HSD will spontaneously reduce MCF-7 cells growth [28].

Therefore, this work is aimed at identifying the molecular descriptors responsible for antibreast cancer activities of selected compounds in Annona muricata seed and studies the nonbonding interactions as well as the actual binding energy between the selected compounds and $3 \alpha$ HSD using docking and molecular dynamics simulation methods, respectively.

\section{Methodology}

2.1. Optimization and Molecular Docking. Five molecular compounds selected from Annona muricata seed were screened against $3 \alpha$-HSD via an in silico method. The crystal structure was retrieved from protein data bank [29] with the accession code 4XO6 [28]. The selected compounds were optimized using Spartan 14 [30]. Also, the docking study was executed using a series of software (Discovery studio 2017, AutoDock Tool 1.5.6, AutoDock Vina 1.1.2, and EduPyMol version 1.7.4.4), and the calculated binding affinities were reported.

This docking study involves the preparation of $3 \alpha-\mathrm{HSD}$ and the studied ligands using PyMol. The binding site in the studied receptor was located using AutoDock Tool, and the docking calculation was performed via AutoDock Vina. More so, the nonbonding interaction was viewed through Discovery studio 2017. The observed grid box was as follows: center $(X=12.444, Y=-0.171$, and $Z=2.943)$ and size ( $X=62, Y=60$, and $Z=74$ ), and the spacing was set to be $1.00 \AA$ A.

The selected compounds from Annona muricata seed are (2S)-2-methyl-4-[(2R, 10R, 13R)-2, 10, 13-trihydroxy-13$[(2 R, 5 R)-5$-[(1R)-1-hydroxytridecyl $]$ oxolan-2-yl $]$ tridecyl $]$ $2 H$-furan-5-one (A), (2S)-2-methyl-4-[(2R, 8R, 13R)-2,8,13trihydroxy-13-[(2R, 5S)-5-[(1S)-1-hydroxytridecyl] oxolan2-yl]tridecyl]-2H-furan-5-one (B), 3-\{2, 13-dihydroxy-13[5-(1-hydroxytridecyl)oxolan-2-yl]-8-oxotridecyl $\}$-4-methyl -2,5-dihydrofuran-2-one (C), (2S)-2-methyl-4-[(2R, $8 R$, $11 R)-2, \quad 8, \quad 11$-trihydroxy-11-[(2R, 5S)-5-[(1S)-1-hydroxypentadecyl] oxolan-2-yl] undecyl]-2H-furan-5-one (D), and 5-(1-hydroxytridecyl)oxolan-2-one (E) (Figure 1).

2.2. ADMET Properties. Physicochemical and absorption, distribution, metabolism, excretion, and the toxicity properties of the studied molecular compounds were examined using admetSAR (http://lmmd.ecust.edu.cn/admetsar1) [31]. In this study, several factors were considered such as Ames test, human intestinal absorption, blood-brain barrier, and Caco-2 cell permeability.

2.3. Molecular Dynamic Simulation Studies. The compound with better binding affinity was selected from the compounds docked, and chloroquine was used as a standard; both were subjected to molecular dynamics simulation on AMBER14 molecular dynamics package [32]. Hydrogen atom was added to the studied complexes using a leap segment of AMBER14. The protein was described with AMBER force field 99SB while the ligand with general AMBER force field [33, 34]. To neutralize the studied complexes before solvation, the required number of counterions was added. The solvation was implemented in a condensed octahedral cell of TIP3P [35] water molecules, and $12 \AA$ was stretched outside the protein on each side.

The complexes were minimized using 5000 frames of steepest descent minimization and 10000 frames of conjugated gradient minimization to eliminate bad atom interactions. The entire systems were heated at $300 \mathrm{~K}$ and 50000 ps. Molecular dynamics simulation was accomplished for the studied complex at $300 \mathrm{~K}$ and $1 \mathrm{~atm}$ via the particle mesh Ewald method [36]. CPPTRAJ module [33] implemented in AMBER14 software was used for molecular dynamics trajectories analysis. 


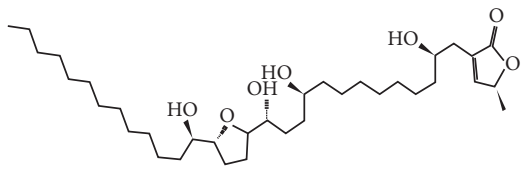

(a)

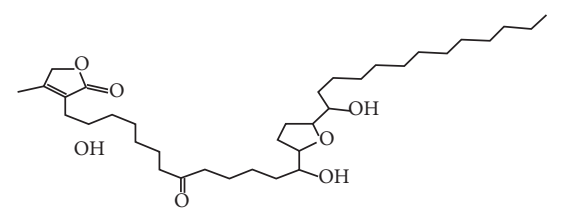

(c)

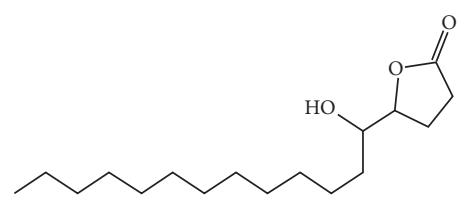

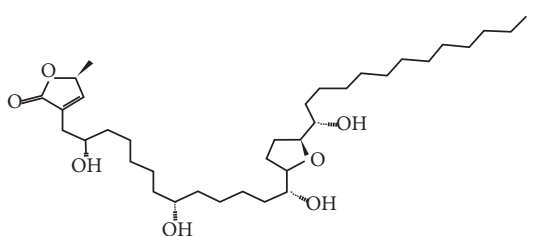

(b)

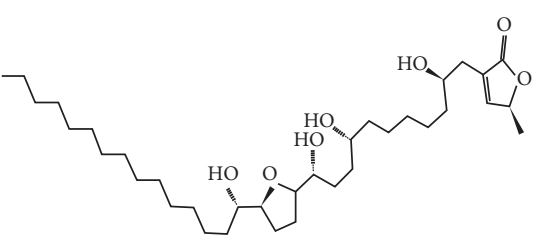

(d)

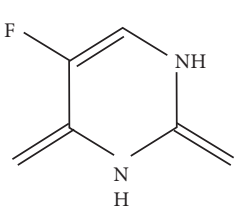

(e)

FIGURE 1: Schematic structure of the studied compounds.

\section{Result and Discussion}

3.1. Density Functional Theory Calculations. Series of molecular descriptors obtained from carefully selected five molecular compounds in Annona muricata seed via density functional theory (DFT) are highest occupied molecular orbital energy $\left(E_{\mathrm{HOMO}}\right)$, lowest unoccupied molecular orbital energy ( $\left.E_{\mathrm{LUMO}}\right)$, dipole moment, energy bandgap, area, volume, polarizability, polar surface area, $\log P$, hydrogen bond donor, and hydrogen bond acceptor (Table 1). In this work, the contribution of dipole moment to the biological activity of studied compounds was observed. According to David et al., it was reported that the presence of dipole moment as a nonbonded interaction in the drug-like compound-protein complex is vital [37]. The accepted range for dipole moment is $3-5 \mathrm{~kJ} / \mathrm{mol}$; thus, compound $\mathbf{E}$ was the only compound that falls within the set standard, so it is expected to have strong nonbonded connections $3 \alpha$-HSD.

Furthermore, the calculated $\log P$ reveals the dissolving capacity of drug-like molecules in lipophilic media [38]. As reported by Meanwell 2011, there may be complications in oral absorption if the calculated $\log \mathrm{P}$ is greater than the standard value for $\log P(\log P \leq 5)$ [36]. Therefore, compound $\mathbf{E}$ is expected to dissolve well in nonaqueous substance and possesses the ability to be administered through the human mouth. More so, the calculated $E_{\mathrm{HOMO}}$ which describes the tendency of drug-like molecules to donate electron to the neighbouring compounds was calculated to be $-6.74(\mathbf{A}),-6.69(B),-6.66(C),-6.75(D)$, and $-7.17(\mathbf{E})$. As shown in Table 1, compound $\mathbf{C}$ is expected to inhibit than other studied compounds [39]. The calculated $E_{\mathrm{LUMO}}$ shows the capability of compounds to accept electrons from nearby molecules [40]. In this regard, compound $\mathrm{C}$ with $-1.15 \mathrm{eV}$ is expected to possess a greater ability to accept electron from the nearby compounds than other studied compounds. Other studied calculated descriptors are displayed in Table 1.

More so, the calculated band gap shows the level of reactivity of compounds with drug potentials. Also, many reports from several researchers have proved that level of reactivity of molecular compounds is a function of lower bandgap; thus, compound $\mathbf{C}$ is expected to react better than other studied compounds [41]. Figure 2 shows the $E_{\mathrm{HOMO}^{-}}$ $E_{\text {LUMo overlay. }}$

Furthermore, chiral information of some stereogenic centers of the studied compounds as displayed in Figure 1 was investigated. In compound $\mathbf{A}$, seven (7) chiral centers were observed ( $4 R$ and $3 S$ ), and seven chiral centers were also observed in compound $\mathbf{B}$ with $7 R$ and $6 R$ as the chiral center was observed in compound $\mathbf{C}$. More so, seven (7) and two (2) chiral centers were detected in compounds $\mathbf{D}$ and $\mathbf{E}$, respectively.

3.2. Prediction of ADMET Properties. Series of obtained ADMET properties using admetSAR server [31] were reported. As shown in Table 2, it was observed that compounds A, B, D, and $\mathbf{E}$ had better human intestinal absorption than the standard used (5FU) except compound $\mathbf{C}$ since drug-like compound with the higher value of human intestinal absorption can be absorbed well in the intestine [42]. The values obtained for the blood-brain barrier for compounds A-E showed that compound $\mathbf{E}$ has a very closer value to that of 5FU. Also, the blood-brain barrier value for compounds A-D falls within the same ranges shown in Table 2. The obtained P-glycoprotein report (nonsubstrate and noninhibitor) for all the studied compounds was similar to those of 5FU. Also, to know the level of mutagenicity of the studied compounds, AMES was considered. Thus, in terms 
TABle 1: Calculated molecular descriptors of the studied compounds.

\begin{tabular}{lccccccccccc}
\hline & $E_{\text {HOMO }}(\mathrm{eV})$ & $E_{\mathrm{LUMO}}(\mathrm{eV})$ & BG & DM & MW & Log $P$ & OVA & PSA & POL & HBD & HBA \\
\hline A & -6.74 & -1.00 & 5.74 & 10.40 & 596.89 & 6.80 & 1.98 & 95.53 & 94.96 & 4 \\
B & -6.69 & -1.14 & 5.55 & 9.82 & 596.89 & 6.80 & 1.98 & 97.35 & 95.03 & 4 & 6 \\
C & -6.66 & -1.15 & 5.51 & 8.38 & 594.87 & 6.95 & 1.98 & 91.71 & 94.65 & 3 & 6 \\
D & -6.75 & -1.00 & 5.75 & 9.90 & 568.83 & 5.96 & 1.94 & 95.53 & 91.99 & 4 & 6 \\
E & -7.17 & -0.03 & 7.14 & 4.86 & 284.44 & 4.54 & 1.61 & 41.29 & 66.74 & 1 & 2 \\
\hline
\end{tabular}

Notes. BG: band gap ( $\left.E_{\mathrm{LUMO}}-E_{\mathrm{HOMO}}\right)$; DM: dipole moment; MW: molecular weight; OVA: ovality; POL: polarizability; HBD: hydrogen bond donor; HBA: hydrogen bond acceptor.
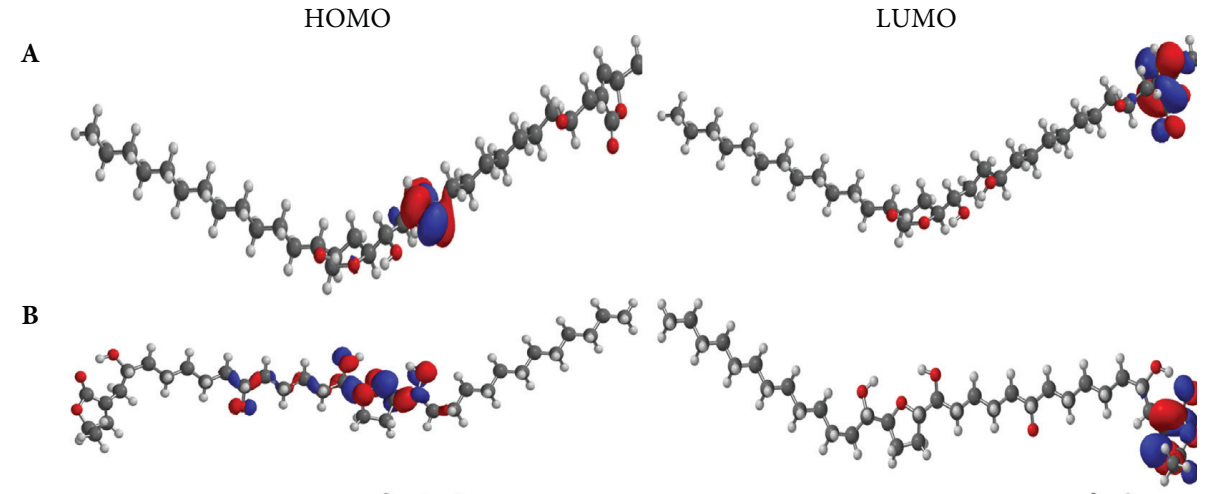

C

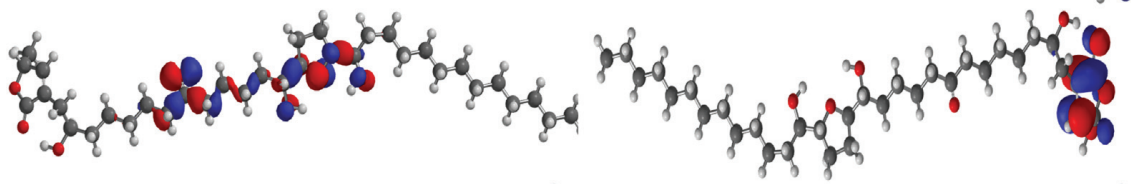

D
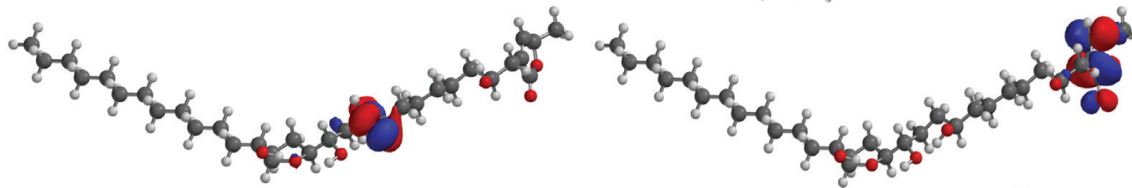

E

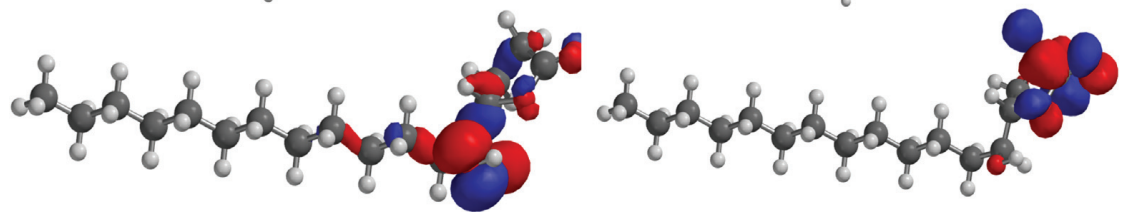

Figure 2: Studied $E_{\text {HOMO }}-E_{\text {LUMO }}$ overlay.

of AMES study, all the studied compounds were negative, and this also correlated with the standard (5FU). As shown in Table 2, all the studied compounds were not carcinogenic and these were similar to the similar used. The calculated cytochrome $\mathrm{P} 450$ showed that it could be inhibited by some of the studied compounds.

3.3. Molecular Docking and Scoring Studies. Series of selected molecular compounds from Annona muricata seed were docked into the active site of $3 \alpha$-HSD to observe the nonbonding interaction that was involved. To establish the accuracy of the engaged docking method, the docked ligand retrieved from the crystal structure of $3 \alpha$-HSD was docked again into the active site of the $3 \alpha$-HSD to determine the similarity of the best conformation of the redocked ligand (i.e., compound with the lowest energy) to the position of the native ligand (Figure 3 ). Hence, the similarity index of the native drug-like compound retrieved from the downloaded receptor to the redocked drug compound was similar to 1 . In this work, the docking method used is observed to be dependable. In this study, it was observed that compound $\mathbf{C}$ has a promising ability to inhibit than other studied compounds. Also, the correlation between the molecular descriptors was observed and it was discovered that $E_{\mathrm{HOMO}}$, $E_{\mathrm{LUMO}}$, and bandgap as displayed in Table 1 greatly contributed to the promising inhibiting activity of compound $\mathbf{C}$ than other studied compounds.

The docking score, as well as the binding affinity obtained for the studied complexes, was calculated to be negative, and this is a sign that the studied compounds could serve as the starting point for developing effective drugs targeting $3 \alpha$-HSD, thereby reducing breast cancer in woman globally. Also, as shown in Table 3, the entire studied compound proved to be more effective than the standard used. The residues involve in each of the conformation are PRO-226, TRP-227, ALA-27, GLU-224, LEU-306, TYR-24, HIS-222, HIS-117, and LYS-270 for A; LYS-270, LEU-268, 


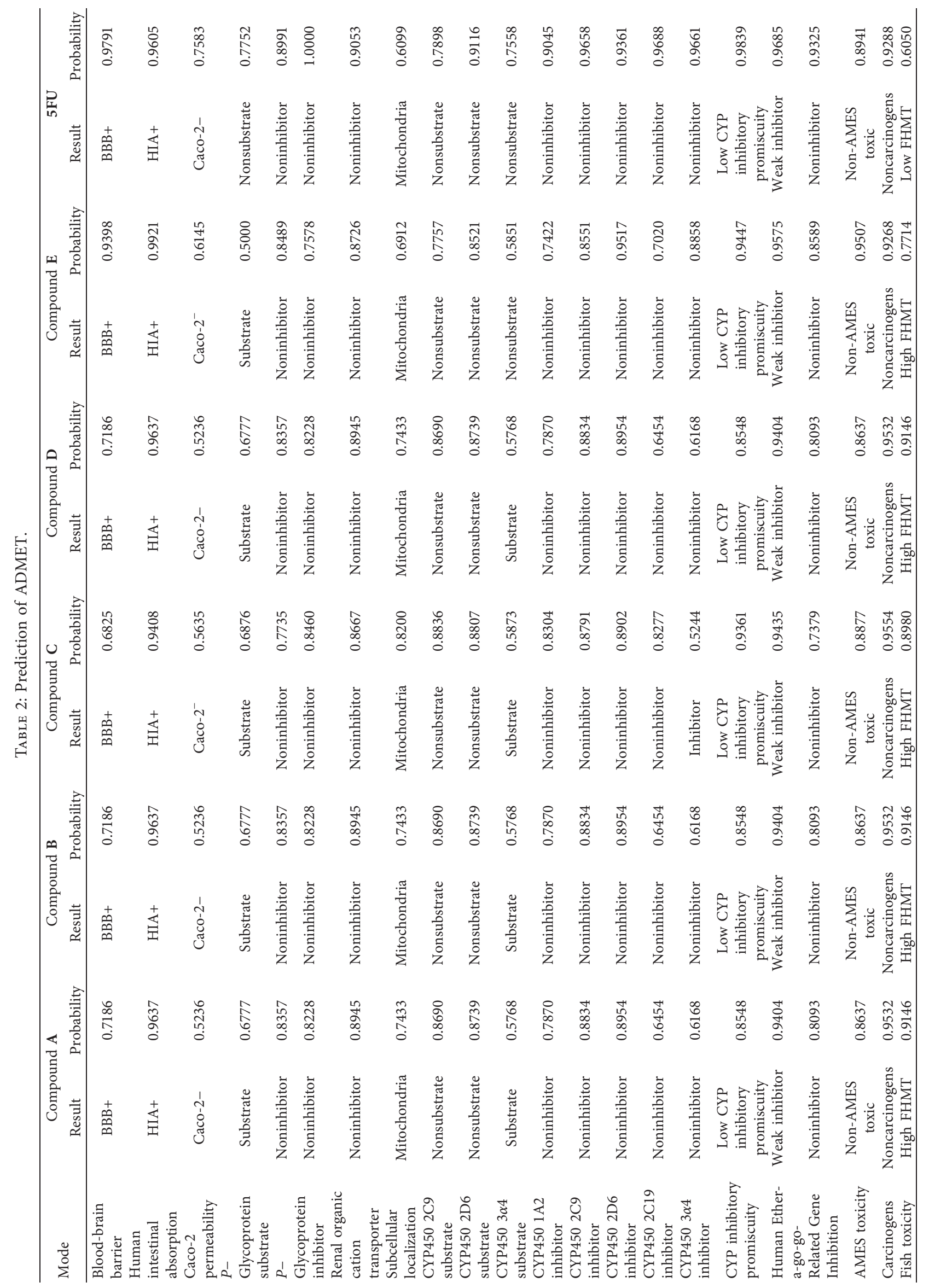




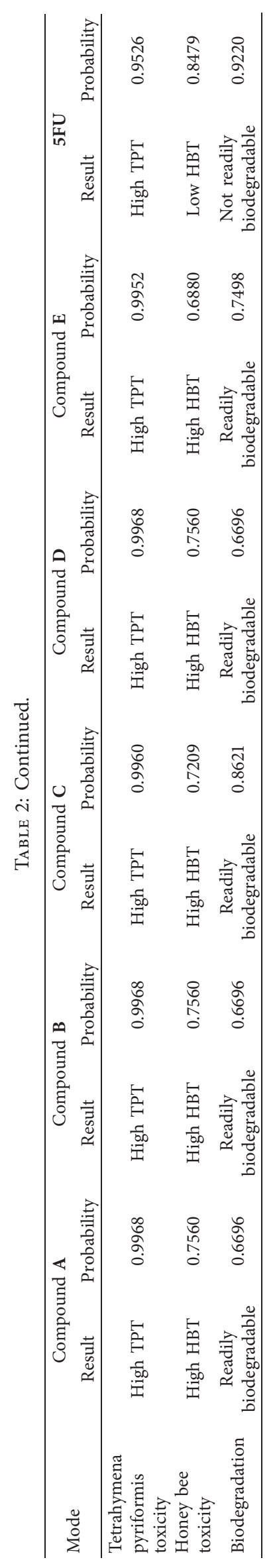




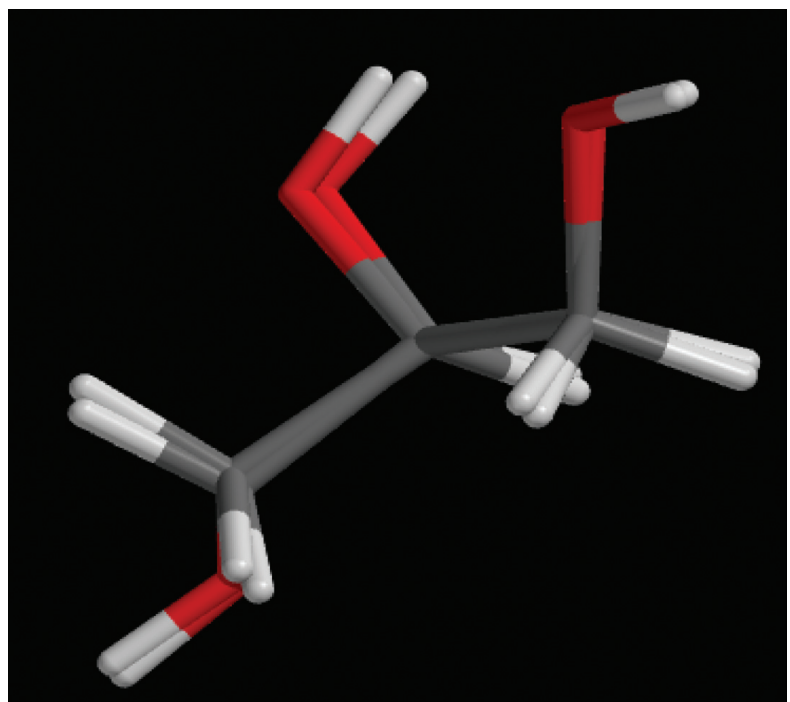

FIGURE 3: Overlay of native drug-like compounds over redocked drug compound.

TABLE 3: Scoring of selected compounds in Annona muricata seed with $3 \alpha$-HSD.

\begin{tabular}{lcc}
\hline & Binding affinity $(\mathrm{kcal} / \mathrm{mol})$ & Amino acid residue \\
\hline A & -7.0 & PRO-226, TRP-227, ALA-27, GLU-224, LEU-306, TYR-24, HIS-222, HIS-117, LYS-270 \\
B & -7.3 & LYS-270, LEU-268, GLY-22, THR-23, ASP-50, TYR-55, GLN-190, TYR-24, VAL-54, VAL 128 \\
C & -7.5 & LYS-270, HIS-222, SER-217, ASP-50, TYR-55, HIS-117, TRP-227, TYR-24, ALA-27 \\
D & -7.1 & PRO-226, TRP-227, LEU-308, LEU-306, TYR-55, TYR-24, HIS-222, LYS-270 \\
E & -7.2 & THR-23, TYR-65, TYR-24, TRP-227 \\
5FU & -5.6 & TYR-55, ASP-50, THR-23, TYR-24, GLY-22 \\
\hline
\end{tabular}

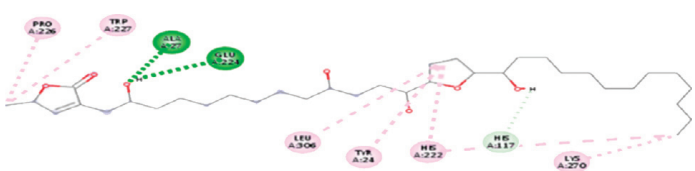

(a)

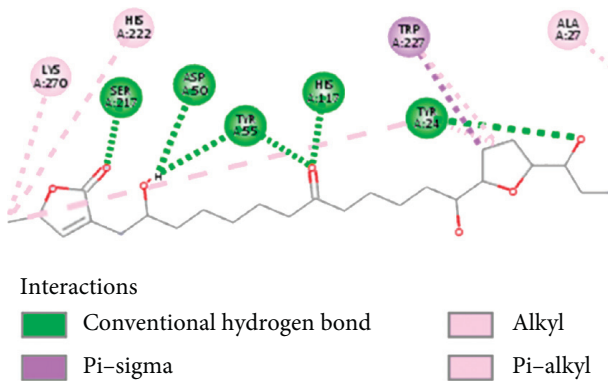

(c)

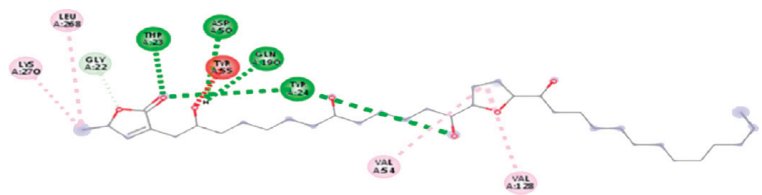

(b)

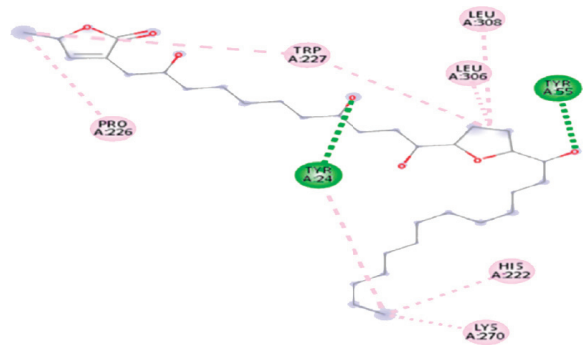

(d)

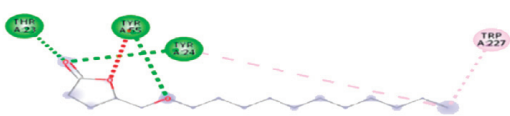

$$
\begin{aligned}
& \text { Interactions } \\
& - \text { Conventional hydrogen bond } \\
& - \text { Unfavorable acceptor-acceptor } \\
& \square \text { Pi-alkyl }
\end{aligned}
$$

(e)

Figure 4: Binding interaction of compounds A, B, C, D, and E with $3 \alpha-H S D$, respectively. 


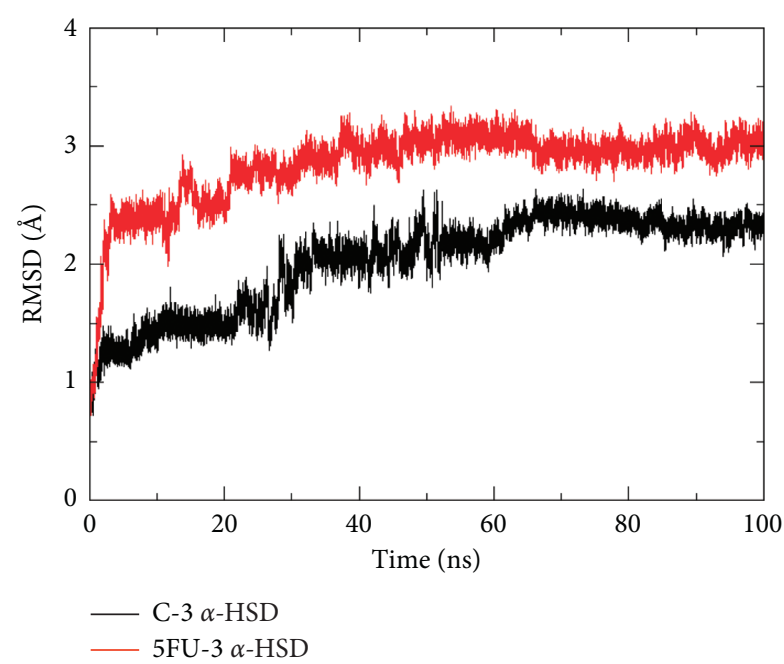

Figure 5: RMSD of compounds C- $3 \alpha$-HSD (black) and 5FU- $3 \alpha$ HSD (red) complexes during the 100 ns MD simulations.

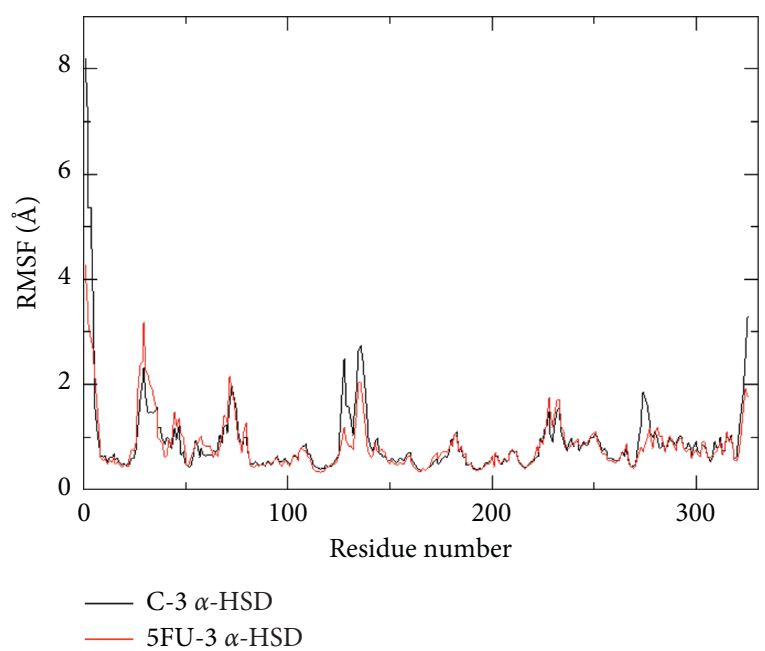

FIGURE 6: RMSF plots of compounds C-3 $\alpha$-HSD (black) and 5FU$3 \alpha$-HSD (red) complexes during the $100 \mathrm{~ns}$ MD simulations.

TABLE 4: Calculated binding free energies and its components for the complexes using the MM-GBSA method extracted from the last 1000 frames of $100 \mathrm{~ns}$.

\begin{tabular}{lccccccc}
\hline Compound & $\Delta E_{\mathrm{vdw}}$ & $\Delta E_{\text {ele }}$ & $\Delta G_{\text {gas }}$ & $\Delta G_{\text {polar }}$ & $\Delta G_{\text {nonpolar }}$ & $\Delta G_{\text {solvation }}$ & $\Delta G_{\text {bind }}$ \\
\hline C & -87.98 & -10.94 & -98.91 & 34.36 & -11.16 & 23.20 & -75.71 \\
5FU & -17.39 & -2.24 & -19.63 & 6.96 & -2.32 & 4.63 & -15.00 \\
\hline
\end{tabular}

The energy components are in $\mathrm{kcal} / \mathrm{mol}$.

GLY-22, THR-23, ASP-50, TYR-55, GLN-190, TYR-24, VAL-54, and VAL-128 for B; LYS-270, HIS-222, SER-217, ASP-50, TYR-55, HIS-117, TRP-227, TYR-24, and ALA-27 for C; PRO-226, TRP-227, LEU-308, LEU-306, TYR-55, TYR-24, HIS-222, and LYS-270 for D; and THR-23, TYR-65, TYR-24, and TRP-227 for E (Figure 4).

\subsection{Molecular Dynamic Simulation Result}

3.4.1. Root of Mean Square Deviation (RMSD). RMSD of $3 \alpha-$ HSD backbone atoms in connection to the initial molecular structure of the studied enzyme in forming complexes with compounds $\mathbf{C}$ and $\mathbf{5 F U}$ during the $100 \mathrm{~ns}$ MD simulation is displayed in Figure 5. This was accomplished to investigate the extent of deviation from the initial structure upon binding and the steadiness of the simulated complexes. Thus, the structures shown in Figure 5 proved that the conformation of the studied complexes was stable and the average RMSD value for compounds C- $3 \alpha-\mathrm{HSD}$ and $5 \mathrm{FU}-3 \alpha-\mathrm{HSD}$ was $1.17 \AA$ and $1.47 \AA$, respectively. The systems were well equilibrated and stable after $60 \mathrm{~ns}$ of the simulation time.

3.4.2. Root of Mean Square Fluctuation. The calculated root mean square fluctuation (RMSF) reveals the flexibility of residues upon binding of the two compounds tested during simulation time. The studied RMSF helped to know the flexibility of the residue present in the studied enzymes during 50000 ps simulation time (Figure 6). As displayed in Figure 5 , there is a similarity in the trend at which the observed residue vacillates; however, little variation was observed between compounds C-3 $\alpha$-HSD and 5FU-3 $\alpha$ HSD. The pattern shown in Figure 5 proved that compound C- $3 \alpha$-HSD exhibited a minor degree of deviation and this shows a higher level of affinity for compound $\mathbf{C}$ to inhibit $3 \alpha$ HSD.

3.4.3. Binding Free Energy. The binding energy for compounds $\mathrm{C}-3 \alpha$-HSD and $5 \mathrm{FU}-3 \alpha$-HSD was calculated using the MM/GBSA method (Table 4).

As shown in Table 4, it was observed that compound $\mathbf{C}$ possesses a better ability to inhibit $3 \alpha$-HSD than the standard used. The calculated free energy includes van der Waal energy $(-87.98 \mathrm{kcal} / \mathrm{mol})$, electrostatic energy $(-10.94 \mathrm{kcal} /$ $\mathrm{mol})$, polar solvation energy $(34.36 \mathrm{kcal} / \mathrm{mol})$, and nonpolar solvation energy $(-11.16 \mathrm{kcal} / \mathrm{mol})$. This specifies that van der Waal energy, electrostatic energy, and nonpolar solvation energy are favourable; however, polar solvation energy was not favourable for the molecular binding of compound C to $3 \alpha$-HSD.

\section{Conclusion}

Breast cancer is an incessantly growing menace worldwide. Despite several ways to combat this deadly disease by many scientists/researchers, its rate of operation among women keeps increasing. Also, $3 \alpha$-HSD contributes to the existence of breast cancer in woman, and inhibiting $3 \alpha$-HSD will impulsively decrease MCF-7 cells growth. In this work, an 
investigation was carried out on Annona muricata L. seed as an anticancer agent via density functional theory, docking study, and molecular dynamics simulation studies. All the studied compounds proved to be active as an antibreast cancer agent. Also, the molecular descriptors that described the antihuman breast cancer activities in the studied compounds and their contribution to antihuman breast cancer of the studied compounds were identified. More so, compound C possesses a better ability to inhibit $3 \alpha$-HSD than other studied compounds and the standard (5FU). Therefore, this study shows the potential ability of Annona muricata L. seed as an effective antibreast cancer agent.

\section{Data Availability}

All data used to support the findings of the study are available from the corresponding author on reasonable request.

\section{Conflicts of Interest}

The authors declare that they have no conflicts of interest.

\section{Acknowledgments}

The authors are grateful to the CHPC (http://www.chpc.ac.za) cluster for the computational resources and Mrs. E. T. Oyebamiji for the assistance in the course of this study.

\section{References}

[1] P. Sejal and K. P. Jayvadan, "A review on miracle fruit of Annona muricata," Journal of Pharmacognosy and Phytochemistry, vol. 5, no. 1, pp. 137-148, 2016.

[2] S. Moghadamtousi, B. Goh, C. Chan, T. Shabab, and H. Kadir, "Biological activities and phytochemicals of Swietenia macrophylla king," Molecules, vol. 18, no. 9, pp. 10465-10483, 2013.

[3] V. C. Ana, M. Efigenia, M. Y. Elhadi, and N. O. Eva, “Annona muricata: a comprehensive review on its traditional medicinal uses, phytochemicals, pharmacological activities, mechanisms of action and toxicity," Arabian Journal of Chemistry, vol. 11, no. 5, pp. 662-691, 2018.

[4] E.-Y. Ko and A. Moon, "Natural products for chemoprevention of breast cancer," Journal of Cancer Prevention, vol. 20, no. 4, pp. 223-231, 2015.

[5] S. Gajalakshmi, S. Vijayalakshmi, and D. V. Rajeshwari, "Phytochemical and pharmacological properties of Annona muricata: a review," International Journal of Pharmacy and Pharmaceutical Sciences, vol. 4, no. 2, pp. 3-6, 2012.

[6] R. Mohd RohaizadMd, A. Roslida, S. Hamizah, and M. Norhafizah, "Annona muricata leaves extracts prevent DMBA/TPA-induced skin tumorigenesis via modulating antioxidants enzymes system in ICR mice," Biomedicine \& Pharmacotherapy, vol. 94, pp. 481-488, 2017.

[7] S. Moghadamtousi, M. Fadaeinasab, S. Nikzad, G. Mohan, H. Ali, and H. Kadir, "Annona muricata (Annonaceae): a review of its traditional uses, isolated acetogenins and biological activities," International Journal of Molecular Sciences, vol. 16, no. 7, pp. 15625-15658, 2015.

[8] N. G. Joseph, O. Rita, and S. B. Lawrence, "Chemical composition, total phenolic content, and antioxidant activities of the essential oils of the leaves and fruit pulp of Annona muricata L. (Soursop) from Ghana," Biochemistry Research International, vol. 2019, Article ID 4164576, 9 pages, 2019.

[9] C. Vijayameena, G. Subhashini, M. Loganayagi, and B. Ramesh, "Phytochemical screening and assessment of antibacterial activity for the bioactive compounds in Annona muricata," International Journal of Current Microbiology and Applied Sciences, vol. 2, no. 1, pp. 1-8, 2013.

[10] A. I. Yaji, H. S. Ab Rahman, M. P. K. Wong, and W. Z. W. Zain, "Potential benefits of Annona muricata in combating cancer: a review," The Malaysian Journal of Medical Sciences: MJMS, vol. 25, no. 1, pp. 5-15, 2018.

[11] L. Betancur-Galvis, J. Saez, H. Granados, A. Salazar, and J. Ossa, "Antitumor and antiviral activity of Colombian medicinal plant extracts," Memórias Do Instituto Oswaldo Cruz, vol. 94, no. 4, pp. 531-535, 1999.

[12] Y. Dai, S. Hogan, E. M. Schmelz, Y. H. Ju, C. Canning, and K. Zhou, "Selective growth inhibition of human breast cancer cells by graviola fruit extract in vitro and in vivo involving down regulation of EGFR expression," Nutrition and Cancer, vol. 63, no. 5, pp. 795-801, 2011.

[13] V. C. George, D. R. N. Kumar, V. Rajkumar, P. K. Suresh, and R. A. Kumar, "Quantitative assessment of the relative antineoplastic potential of the n-butanolic leaf extract of Annona muricata Linn. in normal and immortalized human cell lines," Asian Pacific Journal of Cancer Prevention, vol. 13, no. 2, pp. 699-704, 2012.

[14] L. Valencia, D. L. Muñoz, S. M. Robledo et al., "Actividad tripanocida y citotóxica de extractos de plantas colombianas," Biomédica, vol. 31, no. 4, pp. 552-559, 2011.

[15] Y. Gavamukulya, F. Abou-Elella, F. Wamunyokoli, and H. AEl-Shemy, "Phytochemical screening, anti-oxidant activity and in vitro anticancer potential of ethanolic and water leaves extracts of Annona muricata (Graviola)," Asian Pacific Journal of Tropical Medicine, vol. 7, pp. S355-S363, 2014.

[16] K. P. Shashanka and D. Devananda, "Annona muricata (Linn.) acetogenins as potent anti-breast cancer agents," International Journal of Pharmacognosy \& Chinese Medicine, vol. 4, no. 2, 2020.

[17] M. J. Rieser, J. F. Kozlowski, K. V. Wood, and J. L. McLaughlin, "Muricatacin: a simple biologically active acetogenin derivative from the seeds of Annona muricata (annonaceae)," Tetrahedron Letters, vol. 32, no. 9, pp. 1137-1140, 1991.

[18] F.-E. Wu, L. Zeng, Z.-M. Gu et al., "New bioactive monotetrahydrofuran Annonaceous acetogenins, annomuricin $\mathrm{C}$ and muricatocin C, from the leaves of Annona muricata," Journal of Natural Products, vol. 58, no. 6, pp. 909-915, 1995.

[19] L. Schwedler, G.-R. Jonathan, A.-K. Sommer, W. Ernst, and R. Andreas, "MiRNA-27a sensitizes breast cancer cells to treatment with selective estrogen receptor modulators," The Breast, vol. 43, pp. 31-38, 2019.

[20] S. P. Helmrich, S. Shapiro, L. Rosenberg et al., "Risk factors for breast cancer," American Journal of Epidemiology, vol. 117, no. 1, pp. 35-45, 1983.

[21] L. A. Kaufman, E. Steel, P. Weideman et al., "Consumer and clinician perspectives on personalising breast cancer prevention information," The Breast, vol. 43, pp. 39-47, 2019.

[22] C. M. Gwenna€elle and R. Pedro, "Rationale for immunological approaches to breast cancer therapy," The Breast, vol. 37, pp. 187-195, 2018.

[23] M. Berli_ere, F. Roelants, C. Watremez et al., "The advantages of hypnosis intervention on breast cancer surgery and adjuvant therapy," The Breast, vol. 37, pp. 114-118, 2018. 
[24] G. Ren-Shan, O. H. Dianne, F. C. James, and P. H. Matthew, "Opposing changes in $3 \alpha$-hydroxysteroid dehydrogenase oxidative and reductive activities in rat leydig cells during pubertal development," Biology of Reproduction, vol. 60, pp. 855-860, 1999.

[25] N. Virginie, G. Anne, L. Pierre et al., "Structure of the human 3-hydroxysteroid dehydrogenase type 3 in complex with testosterone and NADP at $1.25-\AA$ resolution," The Journal of Biological Chemistry, vol. 276, no. 45, pp. 42091-42098, 2001.

[26] J. A. Aka, M. Mazumdar, C.-Q. Chen, D. Poirier, and S.-X. Lin, "17 $\beta$-hydroxysteroid dehydrogenase type 1 stimulates breast cancer by dihydrotestosterone inactivation in addition to estradiol production," Molecular Endocrinology, vol. 24, no. 4, pp. 832-845, 2010.

[27] T. M. Penning, Y. Jin, T. L. Rizner, and D. R. Bauman, "Prereceptor regulation of the androgen receptor," Molecular and Cellular Endocrinology, vol. 281, no. 1-2, pp. 1-8, 2008.

[28] Z. Bo, H. Xiao-Jian, W. Xiao-Qiang et al., "Human $3 \alpha$ hydroxysteroid dehydrogenase type 3: structural clues of $5 \alpha$-DHT reverse binding and enzyme down-regulation decreasing MCF7 cell growth," Biochemical Journal, vol. 473, no. 8, pp. 1037-1046, 2016.

[29] H. M. Berman, J. Westbrook, Z. Feng et al., "The protein data bank," Nucleic Acids Research, vol. 28, no. 1, pp. 235-242, 2000.

[30] Wavefunction, Inc., Spartan 14 Program, Wavefunction, Inc., Irvine, CA, USA, 2011.

[31] S. Jie, C. Feixiong, X. You, L. Weihua, and T. Yun, "Estimation of ADME properties with substructure pattern recognition," Journal of Chemical Information and Modeling, vol. 50, no. 6, pp. 1034-1041, 2010.

[32] J. Wang, R. M. Wolf, J. W. Caldwell, P. A. Kollman, and D. A. Case, "Development and testing of a general amber force field," Journal of Computational Chemistry, vol. 25, no. 9, pp. 1157-1174, 2004.

[33] J. Sgrignani, G. Grazioso, M. De Amici, and G. Colombo, "Inactivation of TEM-1 by avibactam (NXL-104): insights from quantum mechanics/molecular mechanics metadynamics simulations," Biochemistry, vol. 53, no. 31, pp. 5174-5185, 2014.

[34] W. L. Jorgensen, J. Chandrasekhar, J. D. Madura, R. W. Impey, and M. L. Klein, "Comparison of simple potential functions for simulating liquid water," The Journal of Chemical Physics, vol. 79, no. 2, pp. 926-935, 1983.

[35] T. Darden, D. York, and L. Pedersen, "Particle mesh Ewald: an $N \cdot \log (N)$ method for Ewald sums in large systems," The Journal of Chemical Physics, vol. 98, no. 12, pp. 10089-10092, 1993.

[36] D. R. Roe and T. E. Cheatham III, "PTRAJ and CPPTRAJ: software for processing and analysis of molecular dynamics trajectory data," Journal of Chemical Theory and Computation, vol. 9, no. 7, pp. 3084-3095, 2013.

[37] A. Khaled, R. Petri, M. Sampo, and P. Olavi, "Metabolism of $\alpha$-Thujone in human hepatic preparations in vitro," Xenobiotica, vol. 41, pp. 101-111, 2011.

[38] N. A. Meanwell, "Synopsis of some recent tactical application of bioisosteres in drug design," Journal of Medicinal Chemistry, vol. 54, no. 8, pp. 2529-2591, 2011.

[39] A. K. Oyebamiji and B. Semire, "Dft-Qsar model and docking studies of antiliver cancer (Hepg-2) activities of 1, 4-diydropyridine based derivatives," Cancer Biology, vol. 6, no. 2, pp. 69-78, 2016.

[40] A. K. Oyebamiji, F. O. Oyedeji, I. A. Adejoro, and B. B. Adeleke, "Anti-fungal activities of 2, 4-dintrophenyl hydrazones derivatives: DFT and docking approaches," Academia Arena, vol. 10, no. 6, pp. 2-16, 2018.

[41] A. K. Oyebamiji and B. Semire, "Studies of 2-[5-(aryloxymethyl)-1, 3, 4-oxadiazol-2-ylsulfanyl] acetic acid derivatives for antibacterial activities via DFT, QSAR and docking approaches," Bulletin of Pharmaceutical Research, vol. 7, no. 3, p. 148, 2017.

[42] O. Mebarka, B. Salah, L. Khaled, D. Ismail, and B. Houmam, "Molecular docking studies and ADMET properties of new 1.2.3 triazole derivatives for anti-breast cancer activity," Journal of Bionanoscience, vol. 12, pp. 26-36, 2018. 